\title{
Impact of non-uniform surface magnetic fields on stellar winds
}

\author{
V. Holzwarth
}

\author{
School of Physics and Astronomy, University of St. Andrews, North Haugh, St. Andrews, Fife KY16 9SS, Scotland, UK \\ e-mail: vrh1@st-andrews.ac.uk
}

Received 22 March 2005 / Accepted 26 May 2005

\begin{abstract}
Observations of active stars reveal highly non-uniform surface distributions of magnetic flux. Theoretical models considering magnetised stellar winds however often presume uniform surface magnetic fields, characterised by a single magnetic field strength. The present work investigates the impact of non-uniform surface magnetic field distributions on the stellar mass and angular momentum loss rates as well as on the effective Alfvénic radius of the wind. Assuming an axial symmetric and polytropic magnetised wind, the approach of Weber \& Davis (1967, ApJ, 148, 217) is extended to non-equatorial latitudes to quantify the impact of latitude-dependent magnetic field distributions over a large range of stellar rotation rates and thermal wind properties. Motivated by recent observational results, the analytically prescribed field patterns are dominated by magnetic flux concentrations at intermediate and high latitudes. The global stellar mass loss rates are found to be rather insensitive to non-uniformities of the surface magnetic field. Depending on the non-uniformity of the field distribution, the angular momentum loss rates deviate in contrast at all rotation rates between $-60 \%$ and $10 \%$ from the Weber \& Davis-values, and the effective Alfvénic radii up to about $\pm 25 \%$. These large variations albeit equal amounts of total magnetic flux indicate that a classification of stellar surface magnetic fields through a single field strength is insufficient, and that their non-uniformity has to be taken into account. The consequences for applications involving magnetised stellar winds are discussed in view of the rotational evolution of solar-like stars and of the observational determination of their mass loss rates using the terminal velocity and ram pressure of the wind. For rapidly rotating stars the latitudinal variation of the wind ram pressure is found to exceed, depending on the actual field distribution on the stellar surface, over two orders of magnitude. The assumption of a spherical symmetric wind geometry may therefore lead to a significant over- or underestimation of the stellar mass loss rate.
\end{abstract}

Key words. stars: winds, outflows - stars: mass-loss - stars: magnetic fields - stars: starspots - stars: rotation magnetohydrodynamics (MHD)

\section{Introduction}

Stars with hot coronae are subject to mass and angular momentum losses due to stellar winds (Parker 1958). In the presence of magnetic fields the angular momentum (AM) loss is significantly enhanced, because the escaping ionised plasma is forced into co-rotation with the star out to distances much larger than the stellar radius (Schatzman 1962). Further to its contribution to the specific AM of the plasma, the stress of bent magnetic field lines adds to the acceleration of the wind by magneto-centrifugal driving. In rapidly rotating stars this inherently latitude-dependent mechanism is expected to dominate over the isotropic thermal driving (Michel 1969).

Whereas early studies of stellar winds assumed uniform or dipolar surface magnetic fields, high-resolution observations of the solar atmosphere now indicate much more complex boundary conditions at the base of the wind. Doppler Imaging (DI) observations of active stars show non-uniform surface brightness distributions, which are ascribed to the presence of magnetic flux in the form of dark spots (Collier Cameron 2001). They show that on stars rotating more rapidly than the Sun magnetic flux is not only located in equatorial regions, but also at intermediate and high latitudes (Strassmeier 2002, and references therein). Although starspots can a priori not uniquely be associated with wind loss regions, they nevertheless identify locations of possibly open magnetic field structures. Whereas spectroscopic DI enables the reconstruction of starspot maps, spectro-polarimetric Zeeman-DI directly confirms the magnetic origin of the dark surface features (Donati et al. 1997). In combination with field extrapolation techniques, the observed surface magnetic field distributions serve as boundary conditions for the reconstruction of coronal magnetic field topologies and thus for the determination of both closed and open field regions (e.g. Jardine et al. 2002). Recent results show that on rapidly rotating stars open magnetic flux is organised in beltlike structures mainly between intermediate and high latitudes (McIvor et al. 2004).

Weber \& Davis (1967, hereafter WD) theoretically investigated the radial structure and properties of the magnetised solar wind assuming that the wind structure determined in the equatorial plane is representative for the entire sphere, implying a monopolar, spherical symmetric field geometry. Parameter studies have been carried out in the framework of this model to investigate the dependence of the AM loss rate on the 
characteristic magnetic field strength, the stellar rotation rate, and thermal wind properties (Belcher \& MacGregor 1976; Yeh 1976). Mestel (1968) and Mestel \& Spruit (1987) considered dipolar surface fields with closed magnetic structures forming "dead zones" at the equator and radial open fields lines further away from the star; Okamoto (1974) extended this approach to different analytical poloidal field structures. A selfconsistent determination of the poloidal field structure requires the solution of non-linear partial differential equations, which represents a formidable aspect of the overall wind problem. Theoretical and numerical investigations including this aspect use semi-analytical techniques based on the separation of variables (Vlahakis \& Tsinganos 1998; Lima et al. 2001; Sauty et al. 2002) or multi-dimensional MHD simulations (Sakurai 1985; Keppens \& Goedbloed 1999, 2000), respectively. They reveal at large distances from the star a rotation-dependent latitudinal deflection of the magnetic field lines toward the symmetry axis as a result of the collimating effect of the azimuthal field component (e.g. Heyvaerts \& Norman 1989; Tsinganos \& Bogovalov 2000; Okamoto 2000). The self-consistent treatments are mainly focused on the (asymptotic) wind structure without going into details about mass and AM losses for different stellar parameters. Owing to the non-linearity of the influence of the poloidal field component on the overall wind structure, the differences between self-consistent methods and simplified approximations are difficult to predict and have to be verified through direct comparisons. Therefore, the results of WD-like approaches should a priori be regarded as estimates within the framework of the applied simplifications.

The approaches of Weber \& Davis (1967) and Mestel \& Spruit (1987) are widely used for the determination of AM loss rates in the course of the rotational evolution of stars (Endal \& Sofia 1981; Collier Cameron \& Jianke 1994; Li 1999). In contrast, Solanki et al. (1997) and Holzwarth \& Jardine (2005) used specific modifications of the WD model to investigate the impact of non-uniform surface fields. To determine mass loss rates of solar-like stars, Wood \& Linsky (1998) and Wood et al. (2002) devised a method using the line-of-sight absorption of the $\operatorname{Ly} \alpha$ emission by surrounding astrospheres, which are formed by the collision between the (magnetised) wind from the star and the local interstellar medium. The size of the astrosphere as well as the amount of absorption depend on the ram pressure of the wind, which is a function of the stellar mass loss and wind velocity. For the determination of the total stellar mass loss rate they assume a constant (i.e. spherical symmetric and independent of the rotation rate and latitude) wind velocity. Based on their results, they formulate an empirical relationship between mass loss rates and coronal X-ray fluxes of solar-like stars.

Motivated by the progress in the determination of stellar surface features and mass loss rates, the present work applies the WD wind model to non-equatorial latitudes to investigate the influence of latitude-dependent surface magnetic fields on the total mass and AM loss rates of cool stars. In contrast to self-consistent treatments of the magnetised wind problem, which are focused on detailed properties of the wind structure, the main objective here is the global qualification and quantification of the influence of observed surface magnetic field distributions in relation to the originally spherical symmetric WD method. The applied wind model represents an efficient way to include the aspect of non-uniform surface magnetic fields in the rotational evolution of solar-like stars and in the observational determination of their mass loss rates.

\section{Model assumptions}

The stellar mass and AM loss rates through latitude-dependent magnetised winds are determined for a solar-like star by applying the WD approach to open magnetic field lines at all latitudes separately (cf. Appendix A). The poloidal components of the magnetic field and the flow velocity are assumed to be radial, neglecting the trans-field component of the (stationary) equation of motion perpendicular to the magnetic field. Retaining the full azimuthal wind dependency, the open field lines form spirals on coni with constant opening angles, whose tips are located in the stellar centre.

The polytropic wind is assumed to be symmetric with respect to both the rotation axis and the equator, enabling a limitation of the analyses to the range of co-latitudes $0<\theta \leq \pi / 2$. The wind solutions are determined through the stellar rotation rate and the magnetic and thermal wind conditions prescribed at a reference level, $r_{0}=1.1 R_{\odot}$, close to the stellar surface. The uniform rotation rates cover the range $\Omega=10^{-6}-5.4 \times$ $10^{-4} \mathrm{~s}^{-1} \approx 0.3-180 \Omega_{\odot}$, that is rotation periods $73 \mathrm{~d}-3 \mathrm{~h}$; the upper limit of the rotation rates corresponds to the case when the co-rotation radius, Eq. (A.12), in the equatorial plane is equal to the reference radius.

\subsection{Thermal and magnetic wind properties}

The wind temperature and (particle) density at the reference level are approximated through solar-like values, that is $T_{0}=$ $2 \times 10^{6} \mathrm{~K}$ and $n_{0}=10^{8} \mathrm{~cm}^{-3}$, respectively. With a mean atomic weight $\mu=\mu_{\odot} \simeq 0.6$, these values imply a gas density of $\rho_{0} \simeq 10^{-16} \mathrm{~g} / \mathrm{cm}^{3}$ and a gas pressure of $p_{0} \simeq 3 \times 10^{-2} \mathrm{dyn} / \mathrm{cm}^{2}$. The entropy change of the wind with increasing distance from the star is quantified through the polytropic index, $\Gamma=1.15$. These values fall in the range of values of similar studies (e.g., $\Gamma \simeq 1.2$ and $T_{0}=2.7 \times 10^{6} \mathrm{~K}$, Weber \& Davis 1967; Sakurai 1985; $\Gamma=1.13$ and $T_{0}=1.5 \times 10^{6} \mathrm{~K}$, Keppens et al. 1995), and have been chosen to ensure that even plasma emanating at high latitudes, where the magneto-centrifugal driving is inherently less efficient, escapes from the star owing to a sufficiently strong thermal driving. The thermal boundary conditions are taken to be independent of both latitude and rotation rate.

The magnetic wind properties are determined through the radial magnetic field component at the reference level. For the uniform Constant Field (CO) distributions the field strengths are taken to be $B_{r, 0}=3,30$, and $300 \mathrm{G}$, corresponding to an (unsigned) open magnetic flux between $\Phi \simeq 2 \times 10^{23-25} \mathrm{Mx}$. The lower limit is of the order of the average solar magnetic field strength, whereas the upper limit is in accordance with spectro-polarimetric observations of rapidly rotating stars, which indicate the existence of field strengths up to two orders of magnitude larger than in the case of the Sun (Donati \& Collier Cameron 1997). 

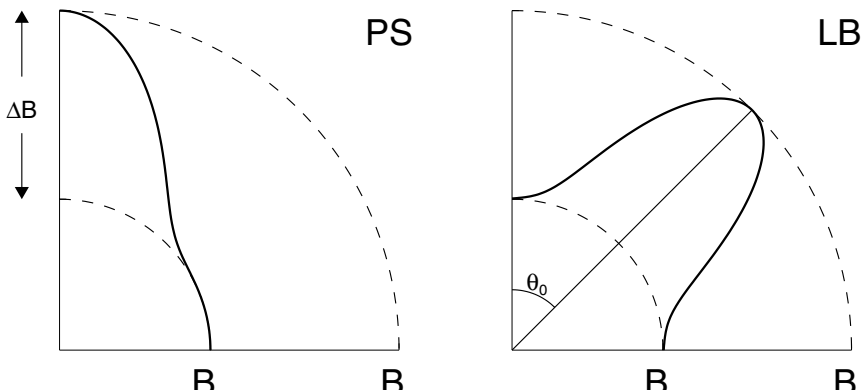

$\mathrm{B}_{<}$

The WD mass loss rate,

$\dot{M}_{\mathrm{WD}}=4 \pi \bar{r}_{\mathrm{A}}^{2} \bar{\rho}_{\mathrm{A}} \bar{v}_{r, \mathrm{~A}}$,

is derived from the assumption that the density, $\bar{\rho}_{\mathrm{A}}$, and radial flow velocity, $\bar{v}_{r, \mathrm{~A}}$, at the Alfvénic point, $\bar{r}_{\mathrm{A}}$, in the equatorial plane are representative for the wind structure at all latitudes, implying $r_{\mathrm{A}}(\theta)=\bar{r}_{\mathrm{A}}, \rho_{\mathrm{A}}(\theta)=\bar{\rho}_{\mathrm{A}}$, and $v_{r, \mathrm{~A}}(\theta)=\bar{v}_{r, \mathrm{~A}}$. All WD values are calculated using the surface averaged field strength, Eq. (1); the determination of wind solutions is outlined in Appendix A.

The AM momentum loss per latitude is

$\mathrm{d} \dot{J}=L F_{\mathrm{M}} \mathrm{d} \sigma=2 \pi \Omega r_{\mathrm{A}}^{4} \rho_{\mathrm{A}} v_{r, \mathrm{~A}} \sin ^{3} \theta \mathrm{d} \theta$,

In the case of non-uniform magnetic fields, latitudedependent distributions are superposed on a constant background field of $B_{<}=3 \mathrm{G}$ (Fig. 1). The peak field strength, $B_{>}=B_{<}+\Delta B$, is chosen so that the surface averaged magnetic field strength,

$\bar{B}_{r, 0}=\int_{0}^{\pi / 2} B_{r, 0}(\theta) \sin \theta \mathrm{d} \theta$,

of each distribution is $30 \mathrm{G}$, to allow for a comparison with the intermediate case of the Constant Field distributions above. Using the analytical form

$B_{r, 0}(\theta)=B_{<}+\Delta B \cdot \cos ^{n}\left(\theta-\theta_{0}\right)$,

the latitude-dependent magnetic field distributions considered here are the

1. Polar Spot model (PS), with $n=8, \theta_{0}=0$, and $\Delta B=$ $243 \mathrm{G}$, and the

2. Latitudinal Belt model (LB), with $n=16, \theta_{0}=45^{\circ}$, and $\Delta B=63.8 \mathrm{G}$.

The first distribution is motivated by observationally determined surface brightness maps of active stars (Strassmeier 2002), which frequently show spot concentrations at high latitudes. The second one is initiated through recent results of coronal magnetic field extrapolations based on ZeemanDoppler Imaging observations of rapid rotators, which reveal a gathering of open magnetic flux also at intermediate latitudes (McIvor et al. 2004).

\subsection{Stellar mass and angular momentum loss rates}

The contribution of a plasma stream to the stellar mass loss rate is given by

$\mathrm{d} \dot{M}=F_{\mathrm{M}} \mathrm{d} \sigma=2 \pi r_{\mathrm{A}}^{2} \rho_{\mathrm{A}} v_{r, \mathrm{~A}} \sin \theta \mathrm{d} \theta$,

with $\mathrm{d} \sigma=\sin \theta \mathrm{d} \theta \mathrm{d} \phi$ being the solid angle occupied by the escaping plasma flow. The mass flux per solid angle, $F_{\mathrm{M}}=$ $\rho v_{r} r^{2}=\rho_{\mathrm{A}} v_{r, \mathrm{~A}} r_{\mathrm{A}}^{2}$, is constant along individual field lines but latitude-dependent, since the plasma density, $\rho_{\mathrm{A}}$, and radial flow velocity, $v_{r, \mathrm{~A}}$, at the Alfvénic point, $r_{\mathrm{A}}$, are functions of the co-latitude. The total stellar mass loss rate is given by

$\dot{M}=\dot{M}_{\mathrm{WD}} \int_{0}^{\pi / 2}\left(\frac{r_{\mathrm{A}}}{\bar{r}_{\mathrm{A}}}\right)^{2}\left(\frac{\rho_{\mathrm{A}}}{\bar{\rho}_{\mathrm{A}}}\right)\left(\frac{v_{r, \mathrm{~A}}}{\bar{v}_{r, \mathrm{~A}}}\right) \sin \theta \mathrm{d} \theta$.

where $L=\Omega\left(r_{\mathrm{A}} \sin \theta\right)^{2}$ is the specific AM along an individual field line (cf. Eqs. (A.3) and (A.5)). The total AM loss rate of the star is then given by

$\dot{J}=\dot{J}_{\mathrm{WD}} \frac{3}{2} \int_{0}^{\pi / 2}\left(\frac{r_{\mathrm{A}}}{\bar{r}_{\mathrm{A}}}\right)^{4}\left(\frac{\rho_{\mathrm{A}}}{\bar{\rho}_{\mathrm{A}}}\right)\left(\frac{v_{r, \mathrm{~A}}}{\bar{v}_{r, \mathrm{~A}}}\right) \sin ^{3} \theta \mathrm{d} \theta$,

with the respective WD value

$\dot{J}_{\mathrm{WD}}=\frac{8 \pi}{3} \Omega \bar{r}_{\mathrm{A}}^{4} \bar{\rho}_{\mathrm{A}} \bar{v}_{r, \mathrm{~A}}=\frac{2}{3} \Omega \bar{r}_{\mathrm{A}}^{2} \dot{M}_{\mathrm{WD}}$.

Latitude-dependent boundary conditions at the base of the stellar wind result in quantities $\left(r_{\mathrm{A}}, \rho_{\mathrm{A}}, v_{r, \mathrm{~A}}\right)$, which are functions of the co-latitude and a priori different from $\left(\bar{r}_{\mathrm{A}}, \bar{\rho}_{\mathrm{A}}, \bar{v}_{r, \mathrm{~A}}\right)$ in the equatorial plane. Even boundary conditions constant at all latitudes cause a deviation of the wind structure from spherical symmetry, since the contribution of the magneto-centrifugal driving to the overall wind acceleration along non-equatorial field lines is smaller than in the equatorial plane.

Studies considering the rotational evolution of stars often determine AM loss rates not through explicit boundary conditions, but by using Eq. (8) in combination with either prescribed or empirical mass loss rates (e.g., Kawaler 1988; Wood et al. 2002), deriving the required Alfvénic radius by more qualitative arguments and/or relationships. The influence of non-uniform field distributions on this approach is quantified through the effective Alfvénic radius,

$r_{\mathrm{A}, \mathrm{eff}}=\bar{r}_{\mathrm{A}}\left(\frac{\dot{J} / \dot{J}_{\mathrm{WD}}}{\dot{M} / \dot{M}_{\mathrm{WD}}}\right)^{1 / 2}$,

which describes the functional relation between the latitudeintegrated mass and AM loss rates in comparison with the WD results.

\section{Results}

\subsection{Reference cases}

The mass loss rates, $\dot{M}_{\mathrm{WD}}$, AM loss rates, $\dot{J}_{\mathrm{WD}}$, and Alfvénic radii, $\bar{r}_{\mathrm{A}}$, determined in the equatorial plane following the approach of Weber \& Davis (1967), are used as reference values. The stellar mass loss rate is controlled by the wind structure in the subsonic flow regime close to the star. Its value depends 


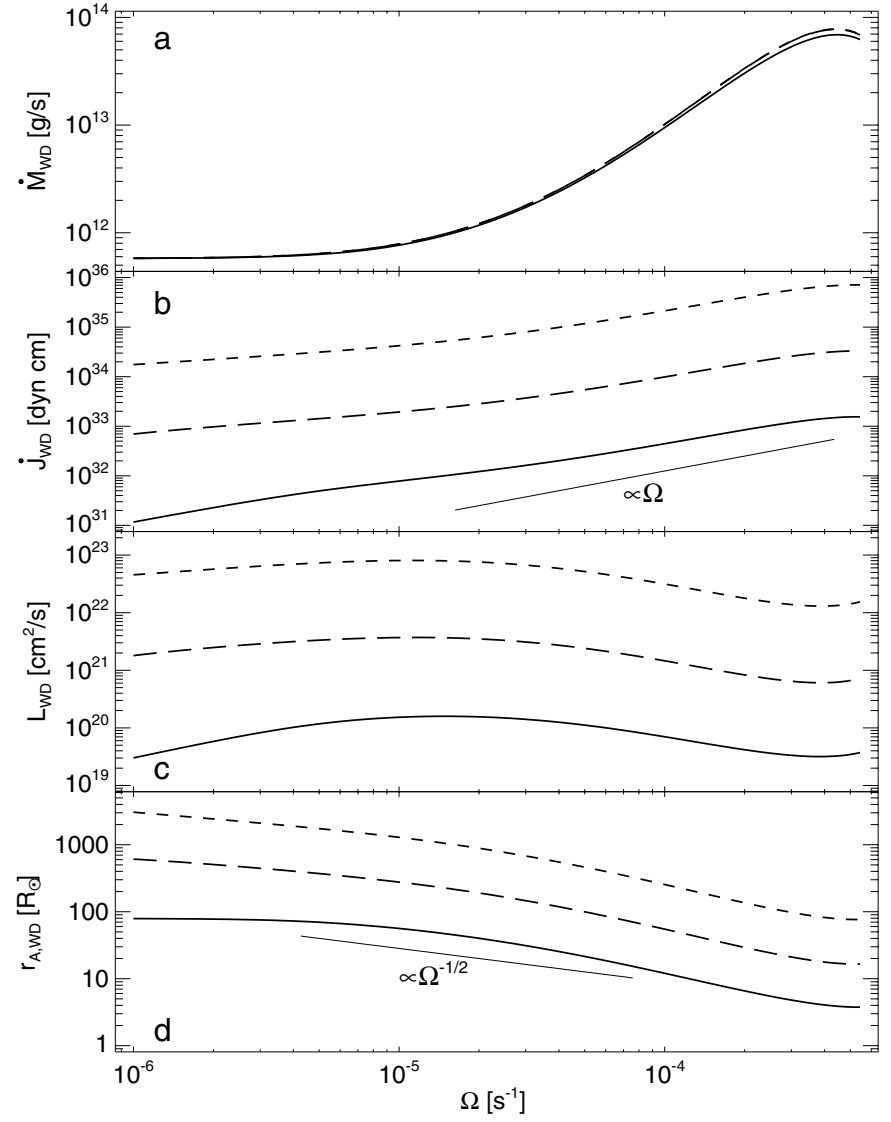

Fig. 2. Reference values determined following the approach of Weber \& Davis (1967), with $\bar{B}_{r, 0}=3 \mathrm{G}$ (solid), 30 G (long dashed), and $300 \mathrm{G}$ (short dashed). a) Mass loss rate, $\dot{M}_{\mathrm{WD}}$; for the two larger field strengths the mass loss rates are quite similar and hardly distinguishable. b) Angular momentum loss rate, $\dot{J}_{\mathrm{WD}}$; the thin line indicates the slope of a function linear in the stellar rotation rate, $\Omega$. c) Specific angular momentum, $L_{\mathrm{WD}}$. d) Alfvénic radius, $r_{\mathrm{WD}}$; the thin line indicates the slope of a function $\propto \Omega^{-1 / 2}$.

significantly on the stellar rotation rate but hardly on the magnetic field strength (Fig. 2a). The increase of the AM loss rate with the rotation rate (Fig. 2b) is due to the strong increase of the mass loss rate, which outbalances minor variations of the specific AM (Fig. 2c). Approximations based on rotationindependent magnetic field strengths imply a linear increase of the AM loss rate, that is $\dot{J} \propto \Omega^{a}$ with $a \simeq 1$, whereas the functional relations here are found to be sub-linear. For rotation rates $\Omega \gtrsim 2 \times 10^{-5} \mathrm{~s}^{-1} \simeq 7 \Omega_{\odot}$ a curve fit yields $a \simeq 0.8$. Below this rotation rate $a \simeq 0.8(3 \mathrm{G}), 0.45(30 \mathrm{G})$ and $0.4(300 \mathrm{G})$, respectively. Due to its explicit linear dependence on the stellar rotation rate, the specific AM increases at slow rotation rates, where the decline of the square of the Alfvénic radius is sublinear (Fig. 2d). At higher rotation rates this decline becomes super-linear and the specific AM consequently smaller, entailing a maximum at intermediate rotation rates.

To verify the impact of non-uniform field distributions with respect to the spherical symmetric WD approach, in the following the results are mainly given in terms of relative deviations, $\delta F=\left(F-F_{\mathrm{WD}}\right) / F_{\mathrm{WD}}$, where $F$ is the mass loss rate, Eq. (4), the AM loss rate, Eq. (7), or the (effective) Alfvénic radius,
Table 1. Reference values determined following the approach of Weber \& Davis (1967), for $\bar{B}_{r, 0}=30 \mathrm{G}$. The solar rotation rate is taken to be $\Omega_{\odot}=2.9 \times 10^{-6} \mathrm{~s}^{-1}$.

\begin{tabular}{rlccc}
\hline \hline & & $\Omega_{\odot}$ & $8 \Omega_{\odot}$ & $48 \Omega_{\odot}$ \\
\hline$(\mathrm{d} \dot{M} / \mathrm{d} \theta)_{\mathrm{WD}}$ & {$\left[10^{11} \mathrm{~g} \mathrm{~s}^{-1} \mathrm{rad}^{-1}\right]$} & 3 & 7 & 91 \\
$F_{\mathrm{M}, \mathrm{WD}}$ & {$\left[10^{10} \mathrm{~g} \mathrm{~s}^{-1} \mathrm{sr}^{-1}\right]$} & 5 & 10 & 145 \\
\hline$(\mathrm{d} \dot{J} / \mathrm{d} \theta)_{\mathrm{WD}}$ & {$\left[10^{32} \mathrm{dyn} \mathrm{cm}^{2} \mathrm{rad}^{-1}\right]$} & 9 & 23 & 101 \\
$L_{\mathrm{WD}}$ & {$\left[10^{20} \mathrm{~cm}^{2} \mathrm{~s}^{-1}\right]$} & 28 & 34 & 11 \\
\hline$v_{\infty, \mathrm{WD}}$ & {$\left[\mathrm{km} \mathrm{s}^{-1}\right]$} & 816 & 2302 & 3298 \\
$p_{\mathrm{w}, \mathrm{WD}}$ & {$\left[10^{18} \mathrm{dyn} \mathrm{sr}^{-1}\right]$} & 4 & 24 & 478 \\
\hline
\end{tabular}

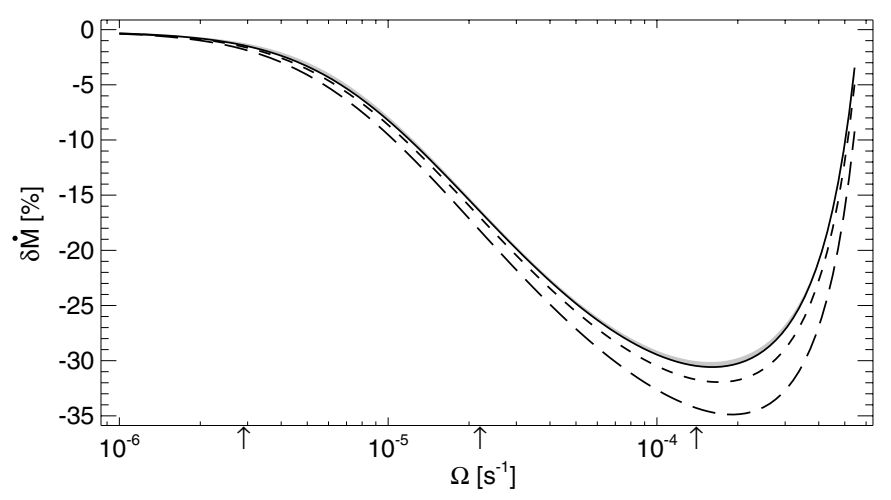

Fig. 3. Relative deviations, $\delta \dot{M}$, of the latitude-integrated mass loss rates from the WD reference values. Constant Field (solid); Polar Spot (long dashed); Latitudinal Belt (short dashed) distribution with $\bar{B}_{r, 0}=30 \mathrm{G}$. The thin grey shaded region indicates the range of values for Constant Field distributions with field strengths between 3-300 G. Arrows mark rotation rates of the latitude-resolved cases shown in Fig. 4.

Eq. (9), respectively. Further quantities have been used to normalise the latitudinal profiles in Figs. 4, 6, and 8; their values are given in Table 1.

\subsection{Mass loss rate}

With increasing rotation rate the latitude-integrated mass loss rates fall short of the WD value, in the domain of rapid rotators up to about $35 \%$ (Fig. 3). The major mass loss originates from the equatorial region, where the magneto-centrifugal driving of the wind is most efficient. However, the mass loss rate per latitude, $\mathrm{d} \dot{M} / \mathrm{d} \theta$, decreases significantly with increasing latitude (relative to the WD values), depending on the rotation rate about two to three orders of magnitude (Fig. 4). This strong decline is caused by the decreasing mass flux in combination with the latitudinal weighting function $\propto \sin \theta$, which takes the smaller surface fraction at higher latitudes into account. In the regime of slow stellar rotation the wind acceleration is caused by the latitude-independent thermal driving, so that the mass flux is virtually constant at all latitudes. In this case the latitudinal variation of $\mathrm{d} \dot{M} / \mathrm{d} \theta$ is dominated by the weighting function. For larger rotation rates the latitudinal decrease of the mass flux strongly enhances the drop of the total mass loss rate. Since the WD approach implies that the equatorial wind structure is 


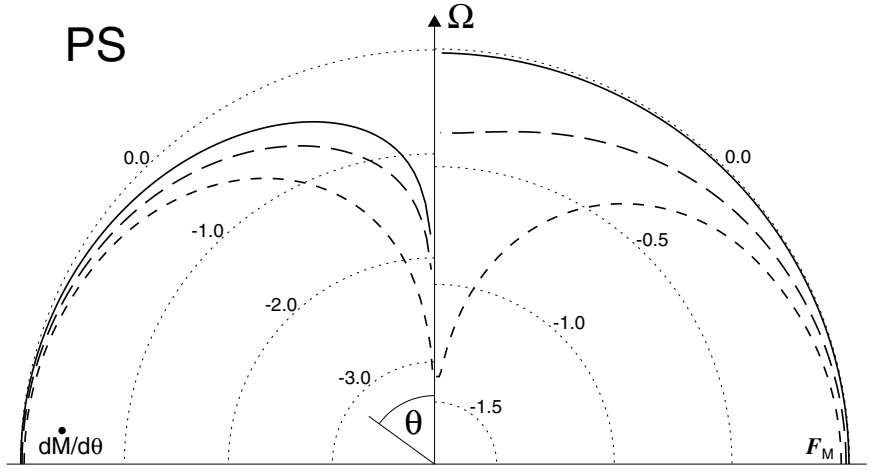

Fig. 4. Left quadrant: relative mass loss rate per latitude, $\lg \left((\mathrm{d} \dot{M} / \mathrm{d} \theta) /(\mathrm{d} \dot{M} / \mathrm{d} \theta)_{\mathrm{WD}}\right)$. Right quadrant: relative mass flux, $\lg \left(F_{\mathrm{M}} / F_{\mathrm{M}, \mathrm{WD}}\right)$. Polar Spot distribution with $\Omega=2.9 \times 10^{-6} \mathrm{~s}^{-1}$ (solid); $2.2 \times 10^{-5} \mathrm{~s}^{-1}$ (long dashed); $1.4 \times 10^{-4} \mathrm{~s}^{-1}$ (short dashed); the respective latitudinal profiles of the Latitudinal Belt and Constant Field distributions are qualitatively very similar and therefore not shown. Note that with $r_{0}^{2} \rho_{0}=$ const. the relation $F_{\mathrm{M}} \propto v_{r, 0}$ holds.

representative for all latitudes, the generalisation of the equatorial mass flux to higher latitudes results in an overestimation of the WD mass loss rate in comparison with the latitude-integrated values.

The deviations of the mass loss rates subject to latitudedependent field distributions are larger than in the case of constant surface fields, but retain the same functional behaviour, with largest deviations occurring at high rotation rates. The Polar Spot yields mass loss rates up to $\sim 10 \%$ smaller than the Constant Field distributions, whereas the Latitudinal Belt falls short only by a few percent. High-latitude magnetic flux concentrations like a Polar Spot imply smaller field strengths at equatorial regions and consequently a drop of the mass flux and mass loss rate per latitude. The Latitudinal Belt possesses at intermediate latitudes in contrast sufficiently large field strengths and mass fluxes to sustain mass loss rates similar to the case of a Constant Field. Since non-equatorial latitudes contribute less to the overall mass loss rate, this localised mass flux surplus can however not balance the reduction of mass loss at the equator.

\subsection{Angular momentum loss rate}

The AM loss rates depend significantly on the stellar rotation rate and magnetic field strength, but even stronger on the latitudinal field distribution (Fig. 5). In the case of constant surface fields, the deviations of the latitude-integrated AM loss rates from the WD values are at small rotation rates moderate, up to about $10 \%$, and field strength-dependent. In contrast, at large rotation rates the values become only marginal and independent of the field strength. In the case of non-uniform field distributions, the integrated AM loss rates can be significantly different despite of equal total amounts of open magnetic flux. In the case of a rapidly rotating star with a Polar Spot the total AM loss rate is about $60 \%$ smaller than the WD value. In the case of a Latitudinal Belt distribution it is about $10 \%$ larger. In contrast to the mass loss rate, where the deviations increase from marginal values at small rotation rates up to a maximum

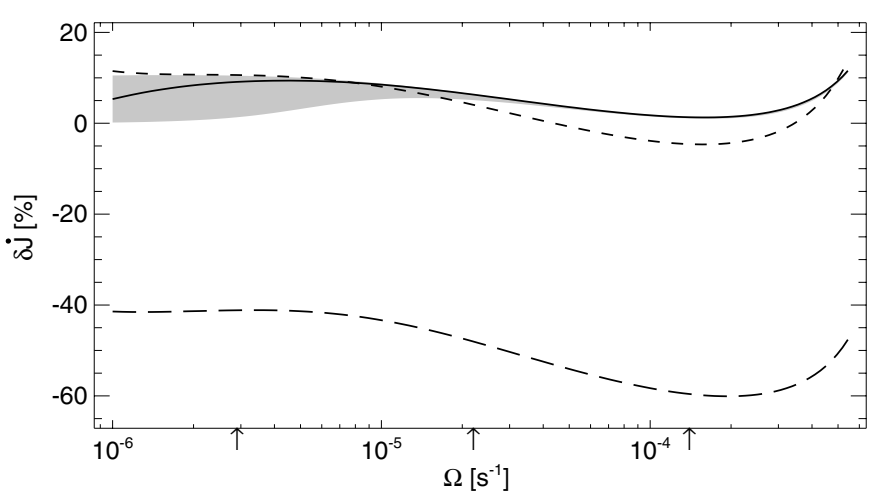

Fig. 5. Relative deviations, $\delta \dot{J}$, of the latitude-integrated angular momentum loss rates from the WD reference values. Constant Field (solid); Polar Spot (long dashed); Latitudinal Belt (short dashed) distribution with $\bar{B}_{r, 0}=30 \mathrm{G}$. The grey shaded region indicates the range of values for Constant Field distributions with field strengths between 3-300 G. Arrows mark rotation rates of the latitude-resolved cases shown in Fig. 6.

in the regime of fast rotation, the deviations of the AM loss rate are significant over the entire range of rotation rates. The functional dependence on the stellar rotation is similar for all field distributions.

The functional behaviour of the AM loss rate is caused by its combined dependence on the mass loss rate (per latitude) and the specific AM, the latter being very susceptible to field strength variations (Fig. 6). The specific AM is a latitudinally weighted function $\propto \sin ^{2} \theta$, due to its dependence on the axial distance of the Alfvénic point. The Alfvénic radius itself increases with latitude and decreases with the rotation rate (cf. Fig. 2), which reflects the changing efficiency of the magnetocentrifugal driving. In the case of uniform magnetic fields, the foreshortening of the geometric lever arm toward high latitudes is therefore countered by the concomitant increase of the Alfvénic radius, and a dependence of the specific AM on the rotation rate opposite to the one of the mass loss rate. The combination of the latter two quantities results in a lateral compensation and therefore in similar $\mathrm{d} \dot{J} / \mathrm{d} \theta$-profiles (Fig. 6, $\mathrm{CO})$. Consequently, the WD approach underestimates the specific AM of non-equatorial regions by assigning the equatorial value of the Alfvénic radius to all latitudes. This underestimation is partly balanced by the overestimation of the mass loss rate, which eventually entails moderate deviations of the latitude-integrated AM loss rates from the WD values.

A Polar Spot distribution yields the largest differences in the latitudinal profile of the specific AM. Although the magnetic field near the pole is very strong, its contribution to the AM loss rate (per latitude) is only average, since at high latitudes the mass loss rates are very small. The reduction of the specific AM at low latitudes causes in contrast a much larger deficit, so that the total AM loss rate is far below the WD value. The Latitudinal Belt distribution also shows a drop of the specific AM around the equator, but since its low-latitude field strengths are larger than in the Polar Spot distribution the differences are in total rather small. The concentration of magnetic flux in a Latitudinal Belt implies high magnetic field strengths at intermediate latitudes. The large specific AM yields in 

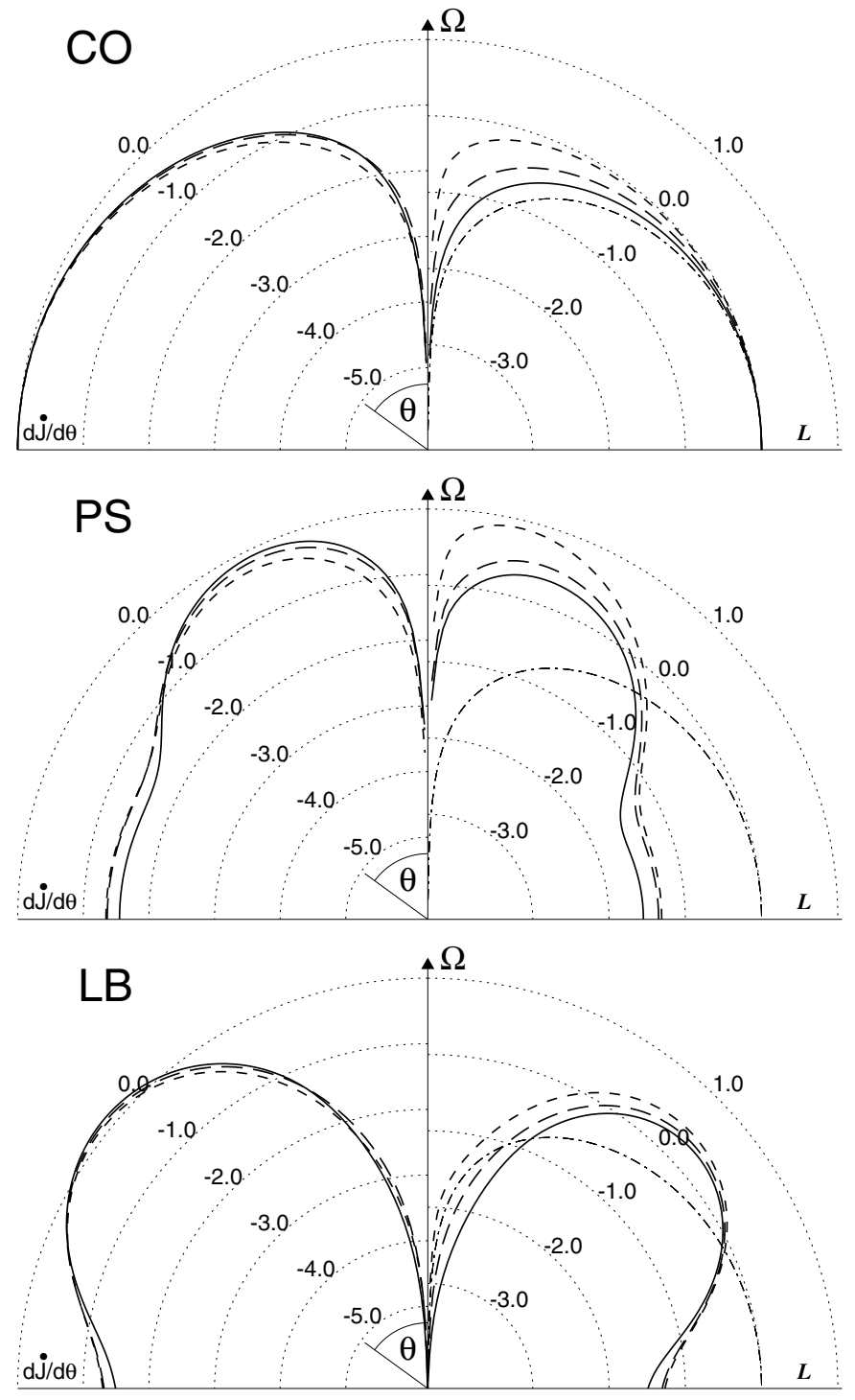

Fig. 6. Left quadrants: relative angular momentum loss rate per latitude, $\lg \left[(\mathrm{d} \dot{J} / \mathrm{d} \theta) /(\mathrm{d} \dot{J} / \mathrm{d} \theta)_{\mathrm{WD}}\right]$. Right quadrants: relative specific angular momentum, $\lg \left[L / L_{\mathrm{WD}}\right]$. Constant Field $(C O)$; Polar Spot $(P S)$; Latitudinal Belt $(L B)$ distribution with $\bar{B}_{r, 0}=30 \mathrm{G}$ and $\Omega=$ $2.9 \times 10^{-6} \mathrm{~s}^{-1}$ (solid); $2.2 \times 10^{-5} \mathrm{~s}^{-1}$ (long dashed) $; 1.4 \times 10^{-4} \mathrm{~s}^{-1}$ (short dashed). The dashed-dotted lines show the weighting function $\propto \sin ^{2} \theta$; since $L \propto r_{\mathrm{A}}^{2} \sin ^{2} \theta$, the differences between this line and the solid/dashed lines are caused by the latitudinal variation of the Alfvénic radius.

combination with the substantial mass loss rates AM loss rates (per latitude), which are even larger than in the reference case.

\subsection{Effective Alfvénic radius}

For the cases considered here, the deviations of the effective Alfvénic radius from the WD values are within about $\pm 25 \%$ (Fig. 7). For a Constant Field or Latitudinal Belt distribution the effective Alfvénic radius is larger than the WD value; at small rotation rates the difference is only marginal, but in the case of rapidly rotating stars it reaches a maximum of $\sim 20 \%$. The Polar Spot distribution, in contrast, yields effective Alfvénic

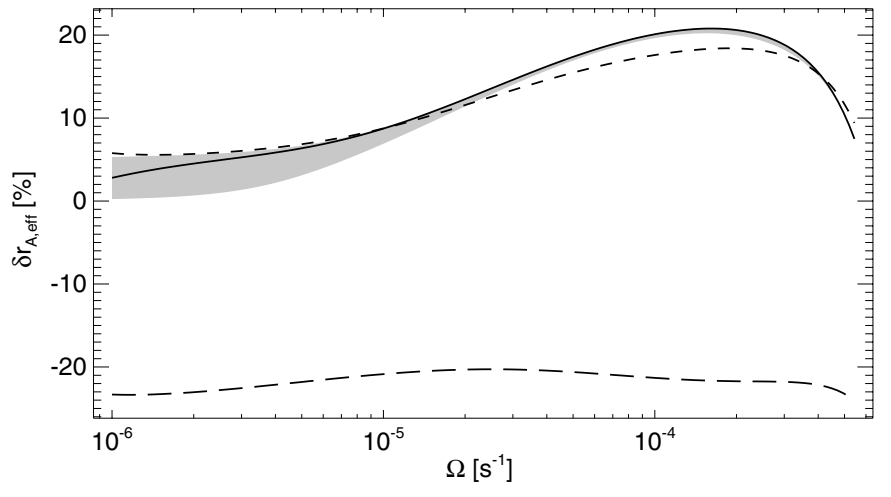

Fig. 7. Relative deviations, $\delta r_{\mathrm{a}, \text { eff }}$, of the effective Alfvénic radii from the WD reference values. Constant Field (solid); Polar Spot (long dashed); Latitudinal Belt (long dashed) distribution with $\bar{B}=30 \mathrm{G}$. The grey shaded region indicates the range of values for Constant Field distributions with field strengths between 3-300 G.

radii which are for all rotation rates nearly $25 \%$ smaller than the reference values.

\subsection{Terminal wind velocity and ram pressure}

The ram pressure, $p_{\mathrm{w}}$, exerted by a magnetised stellar wind far away from the star is approximately proportional to the product of its mass flux (cf. Fig. 4) and terminal wind velocity, $v_{\infty}$. The latitudinal profile of the latter reflects the strong dependence of the magneto-centrifugal driving on the magnetic field distribution and on the stellar rotation rate (Fig. 8). For small field strengths and/or rotation rates this driving mechanism is rather inefficient and the terminal wind velocity thus small. In the case of slowly rotating stars, the latitudinal variation of the ram pressure is found to be within a factor of $\sim 3$ comparable with the WD value, $p_{\mathrm{w}, \mathrm{WD}} \propto \dot{M}_{\mathrm{WD}} v_{\infty, \mathrm{WD}}$. At high rotation rates, however, the latitudinal variation depends significantly on the latitude-dependent magneto-centrifugal driving of the wind, and consequently on the actual magnetic field distribution. In case of a Polar Spot the wind acceleration at high latitudes is supported by strong magnetic fields. There the large terminal velocities make up for smaller mass fluxes, so that the resulting latitudinal variation of the ram pressure is about one order of magnitude. In case of the Latitudinal Belt distribution, for which the magnetic flux is concentrated at intermediate latitudes, the terminal wind velocities at high latitudes become very small and the difference of the ram pressure between intermediate and polar latitudes very large, here over two orders of magnitude.

\section{Dependence on thermal wind properties}

The winds considered here are accelerated through thermal and magneto-centrifugal driving. The latter mechanism is in contrast to the isotropic thermal driving inherently rotationand latitude-dependent. The fractional contribution of each driving mechanism to the overall wind acceleration determines the emphasise of its latitude-dependent character, which consequently changes with the stellar rotation rate. A priori, 

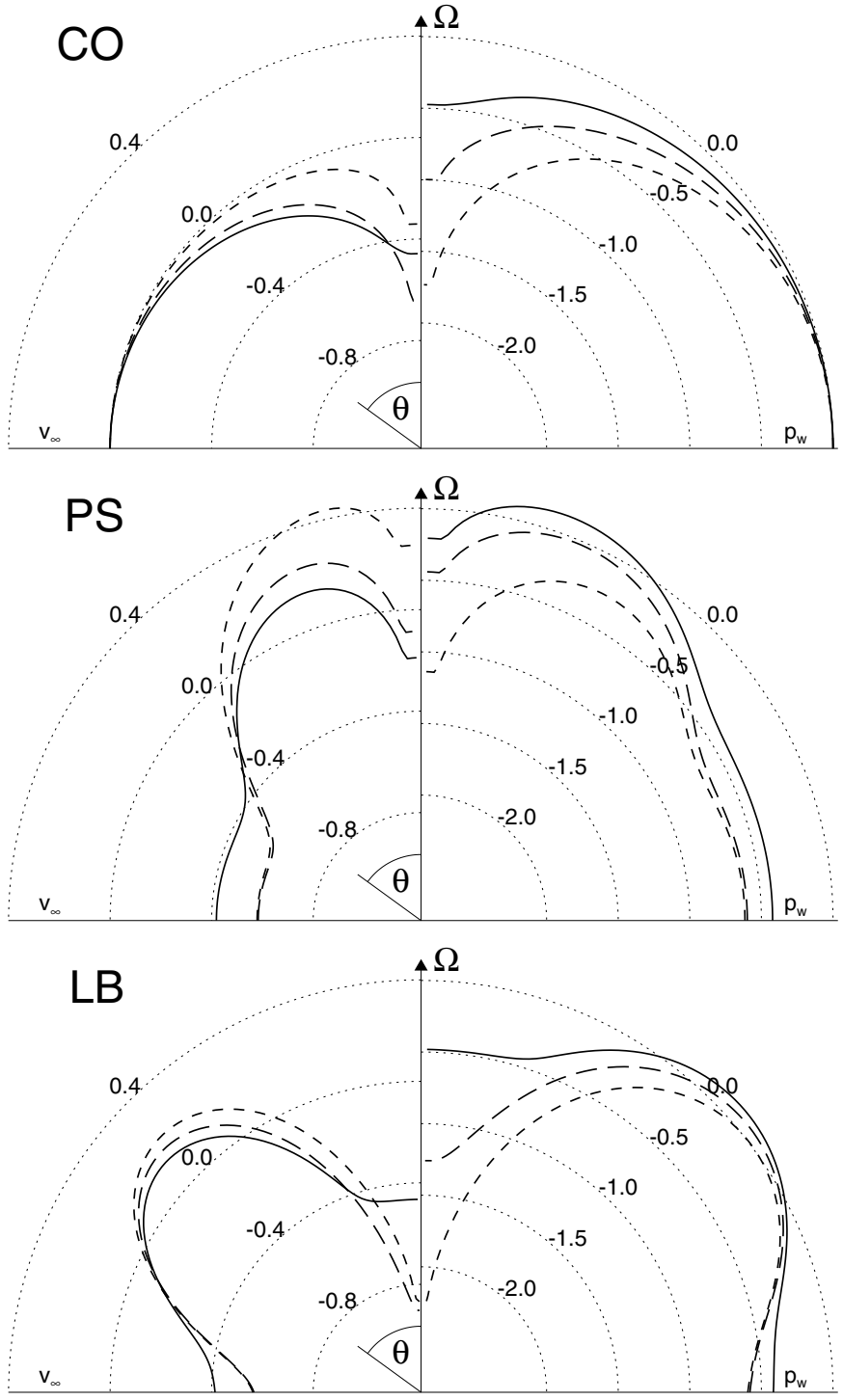

Fig. 8. Left quadrants: relative terminal wind velocity, $\lg \left(v_{\infty} / v_{\infty, \mathrm{WD}}\right)$. Right quadrants: relative wind ram pressure, $\lg \left(p_{\mathrm{w}} / p_{\mathrm{w}, \mathrm{WD}}\right)$. Constant Field (CO); Polar Spot (PS); Latitudinal Belt $(L B)$ distribution with $\bar{B}_{r, 0}=30 \mathrm{G}$ and $\Omega=2.9 \times 10^{-6} \mathrm{~s}^{-1}$ (solid) $; 2.2 \times 10^{-5} \mathrm{~s}^{-1}$ (long dashed); $1.4 \times 10^{-4} \mathrm{~s}^{-1}$ (short dashed).

the thermal driving complies better with the basic WD assumption of a spherical symmetric wind geometry than the latitudedependent magneto-centrifugal driving. To quantify the impact of the thermal wind properties on the previous results, each one of the thermal parameters is successively changed, while the others are kept constant; the parameter ranges are $T_{0}=$ $(1.7-2.5) \times 10^{6} \mathrm{~K} ; n_{0}=10^{-7}-10^{-9} \mathrm{~cm}^{-3} ; \Gamma=1.05-1.175$.

The mass loss rates of rapidly rotating stars, whose outflows are dominated by the magneto-centrifugal driving, are generally less susceptible to changes of the thermal wind properties than those of slow rotators. Higher temperatures and/or stronger heating (i.e. smaller polytropic indices) of the wind shift the emphasise of its acceleration from the magnetocentrifugal to the thermal driving (Fig. 9, top row). The transition between the regimes of thermally controlled and rotationally controlled winds thus occurs at somewhat higher rotation rates. Since the thermal driving is taken to be isotropic, the integrated mass loss rate becomes in turn less susceptible to latitudinal variations of the magnetic field, so that the relative deviations, $\delta \dot{M}$, are smaller. For cooler wind temperatures the effect is vice versa; the mass loss rates are up to $40 \%$ smaller than the WD reference values, with considerable deviations even at small rotation rates. Tenuous winds with lower densities result in smaller deviations of the mass loss rate than more substantial outflows. Thereby, magnetic field concentrations at high latitudes entail a larger susceptibility of the mass loss rate to variations of the wind density than field concentrations at lower latitudes.

The impact of the thermal wind properties on the AM loss rate is rather complex and cannot easily be described in simple terms (Fig. 9, middle row). The specific AM along an open field line depends on the axial distance of the location where the Alfvénic Mach number $(\propto v \sqrt{\rho} / B)$ is unity. Therefore, the smaller the magnetic field strength, or the larger the wind density and/or flow velocity, the closer to the star the Alfvénic point will be located. For higher wind temperatures the specific AM is therefore smaller. But since this decrease is outbalanced by the concomitant increase of the mass loss rate, the resulting AM loss rates are usually larger than for cooler winds. For the thermal wind parameters considered here, the $\delta \dot{J}$-deviations have magnitudes roughly similar to the standard cases (thick solid lines in Fig. 9), although the individual functional behaviour can be quite different. In particular at small rotation rates the deviations are susceptible to the thermal wind properties, showing a rather non-linear behaviour.

Owing to its definition, Eq. (9), the effective Alfvénic radius reflects the functional dependence of both the mass and AM loss rates. Since the deviation of the mass loss rate is typically larger than the deviation of the AM loss rate, the functional dependence of $\delta r_{\mathrm{A} \text {,eff }}$ is less complex than $\delta \dot{J}$; an exception is the case of the Polar Spot distribution, for which the impact on the AM loss rate is very large. The dependence on the wind density is generally rather small, whereas cooler (hotter) winds are found to entail larger (smaller) deviations from the WD values. The same is respectively true for the dependence on the wind heating.

\section{Discussion}

The non-uniform field distributions considered here are motivated through observations of rapidly rotating stars, which frequently indicate the occurrence of magnetic fields up to polar latitudes. Theoretical models explain these high-latitude features in terms of the pre-eruptive poleward deflection of magnetic flux by the Coriolis force (Schüssler \& Solanki 1992), and/or its post-eruptive poleward transport by meridional motions (Schrijver \& Title 2001). The approach of Weber \& Davis (1967, WD) in contrast implies a split monopole (i.e. spherically symmetric) geometry of the magnetic field and wind structure, which results in an overestimation of the mass flux and an underestimation of the specific AM at high latitudes. The latitude-integrated mass and AM loss rates subject to nonuniform surface fields show consequently large deviations from the WD approach. 


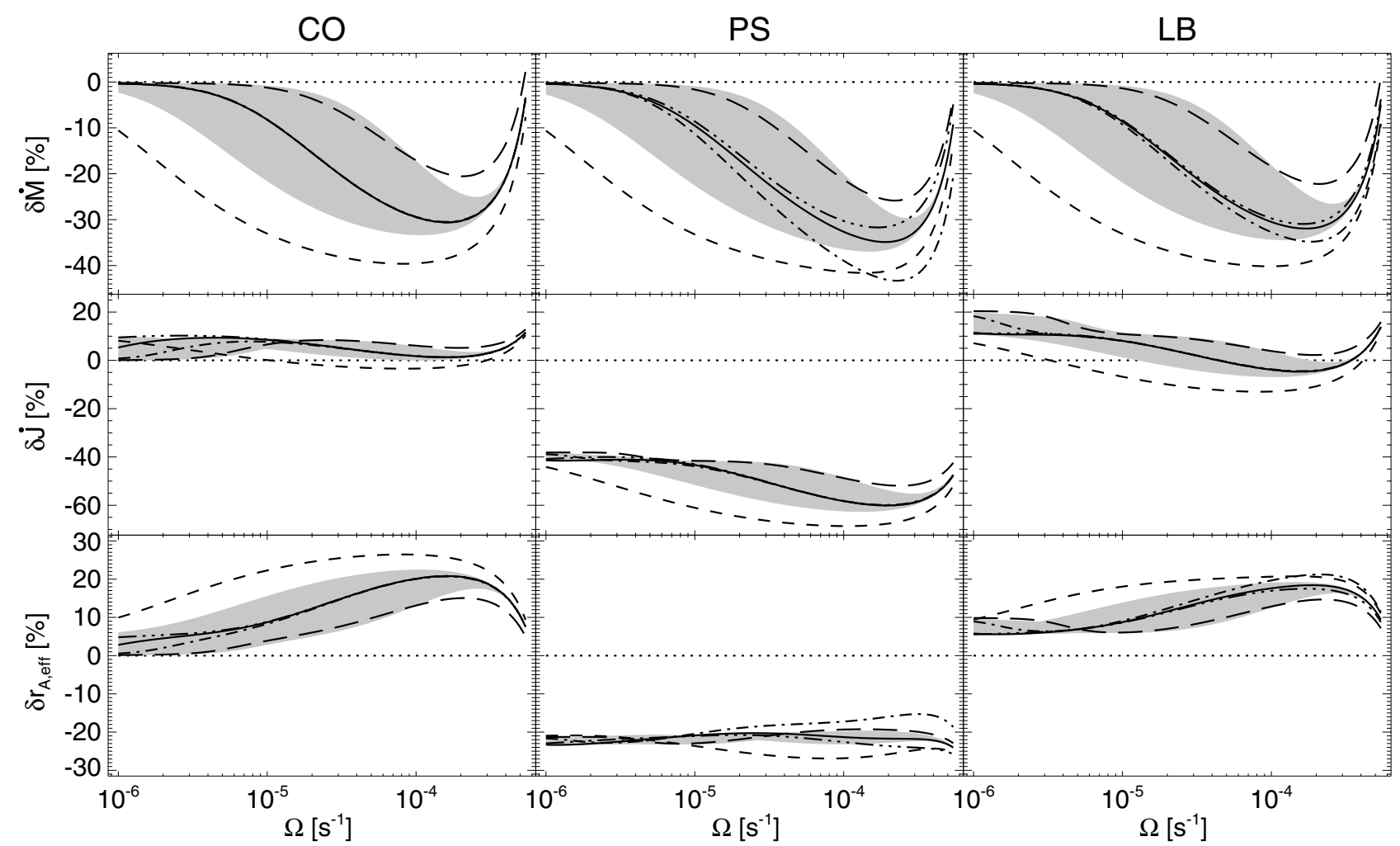

Fig. 9. Relative deviations of the mass loss rate (top), the AM loss rate (middle), and the effective Alfvénic radius (bottom) for the Constant Field (CO); Polar Spot (PS); Latitudinal Belt $(L B)$ distribution. The thermal properties at the base of the wind are $\Gamma=1.15$ and $T_{0}=2 \times 10^{6} \mathrm{~K} \equiv$ $T_{\text {ref }}, n_{0}=10^{8} \mathrm{~cm}^{-3} \equiv n_{\text {ref }}($ solid $) ; T_{0}=2.5 \times 10^{6} \mathrm{~K}, n_{0}=n_{\text {ref }}$ (long dashed) $; T_{0}=1.7 \times 10^{6} \mathrm{~K}, n_{0}=n_{\text {ref }}$ (short dashed) $; T_{0}=T_{\text {ref }}, n_{0}=10^{9} \mathrm{~cm}^{-3}$ (dashed-dotted); $T_{0}=T_{\text {ref }}, n_{0}=10^{7} \mathrm{~cm}^{-3}$ (dashed-triple dotted). The grey shaded regions indicate the range of values for polytropic indices $\Gamma=1.05-1.175$ and $T_{\text {ref }}, n_{\text {ref }}$; the impact of a lower polytropic index is thereby comparable with a higher wind temperature. The (surface averaged) magnetic field strength is in all cases $\bar{B}_{r, 0}=30 \mathrm{G}$.

The deviations of the mass loss rate are largest (up to $30-35 \%$ ) for rapidly rotating stars, since in this regime the dominance of the latitude-dependent magneto-centrifugal driving causes large asymmetries in the mass flux between the equator and the pole. The dependence of this quantity on the field strength is small, so that a redistribution of magnetic flux from equatorial to intermediate or high latitudes hardly changes its latitudinal pattern. The mutual deviations of the mass loss rates between different non-uniform flux distributions are here $\lesssim 10 \%$.

Since the functional dependencies of the mass flux and of the specific AM on the rotation rate follow opposite trends, their combined effects partly balance, which makes the deviation of the AM loss rate from the WD value nearly rotationindependent. Non-uniform fields, on the other side, impose considerable variations on the latitudinal distribution of the specific AM, which are inherited by the AM loss rate causing deviations between $-60 \%$ and $10 \%$. Typically, the stronger the concentration of magnetic flux at higher latitudes, the smaller the AM loss rate. However, for flux concentrations in a Latitudinal Belt at intermediate latitudes the AM loss rate is found to be larger than the WD value, because the higher magnetic field strengths outbalance the foreshortening of the geometric lever arm.

The effective Alfvénic radius reflects the individual properties of the mass and AM loss rates; its deviation form the
WD value are here found to be within about $\pm 25 \%$. Owing to the quadratic dependence on the Alfvénic radius, the AM loss rate can thus deviate up to $\pm 50 \%$ from the WD values, if it is determined in terms of an empirical mass loss rate. The largest uncertainties in the AM loss rate caused by disregarding the actual non-uniformity of surface magnetic fields are likely to occur in the case of rapid stellar rotation, when magnetic flux is gathering at high latitudes.

Both higher wind temperatures and densities are found to increase the mass loss rate, in agreement with previous results (Mestel 1968; Yeh 1976). With increasing wind temperature (or heating) the isotropic thermal driving becomes stronger, and the importance of the magneto-centrifugal driving and of nonuniform field distributions weaker. The transition from thermally to rotationally controlled winds is thus shifted to higher stellar rotation rates. Vice versa, the mass loss rates of cool winds are more susceptible to non-uniformities of the surface magnetic field. The AM loss rates as well as the effective Alfvénic radii show a more complex dependence on the thermal wind properties. The order of magnitude of their deviations from the respective WD values remains roughly unaffected, but the functional behaviours change in complex ways. In accordance with the solar paradigm, high coronal temperatures are ascribed to enhanced magnetic activity, which is found to increase with the stellar rotation rate (Montesinos et al. 2001). Consequently, one has to assume that in rapidly rotating 
stars the structuring of stellar winds arising from the latitudedependent magneto-centrifugal driving and from non-uniform surface magnetic fields is diluted by high wind temperatures, which in turn fortify the isotropic thermal driving.

In this work, the range of magnetic field strengths of the non-uniform field distributions is bracketed through the low- and high-field strength cases of uniform surface fields. However, as mentioned above, their individual loss rates are significantly different. According to this, neither the surface averaged, nor the maximum, nor the minimum field strength enable an accurate characterisation of stellar magnetic fields. In contrast, the AM loss rates of different non-uniform field distributions can cover a large range of values, although their total magnetic flux through the stellar surface is the same. This has important consequences for studies considering the rotational history of stars and the distribution of their rotation rates. By investigating the impact of non-uniform surface magnetic fields on the rotational evolution of stars, Holzwarth \& Jardine (2005) show that concentrations of magnetic flux at high latitudes cause rotational histories which deviate up to $200 \%$ from those obtained following the WD approach. We find that this impact is large enough to mimic deviations of the dynamo efficiency from linearity up to about $40 \%$, and dynamo saturation limits at about 35 times the solar rotation rate (see also Solanki et al. 1997).

Wood \& Linsky (1998) and Wood et al. (2002) analysed the Ly $\alpha$ absorption from the hot $\mathrm{H}$ I wall surrounding astrospheres to determine mass loss rates of solar-like stars. The amount of absorption scales roughly with the square root of the ram pressure of the associated (magnetic) stellar wind, which is in turn a function of the mass loss and wind velocity. To convert the ram pressure into mass loss rates they assume a unique wind velocity $\left(v_{\mathrm{w}}=400 \mathrm{~km} \mathrm{~s}^{-1}\right)$, taken to be isotropic and independent of the stellar rotation rate. Based on their results they determine an empirical relation between the mass lass rates of cool stars and their X-ray surface flux. In the framework of the present wind model the latitudinal profiles of the terminal wind velocity and ram pressure are however found to depend considerably on the stellar rotation rate. For slow rotators the ram pressure varies within a factor of $\sim 3$ with latitude. For rapidly rotating stars, in contrast, the latitudinal variation can rise to over two orders of magnitude. The latitudinal profile of the ram pressure in addition depends strongly on the non-uniformity of the surface magnetic field. If most of the open magnetic flux is located near the poles, then the strong magnetic fields there sustain the magneto-centrifugal driving at high latitudes despite the less efficient centrifugal forces close to the stellar axis of rotation; in this case the latitudinal variation of the ram pressure is about one order of magnitude. If most of the stellar magnetic flux is concentrated at intermediate latitudes, then the polar magnetic field and wind acceleration will in contrast be very weak. Since the strong magneto-centrifugal driving at intermediate latitudes entails high mass fluxes and wind velocities, the difference of the ram pressure with respect to the polar value is over two orders of magnitude. The wind ram pressure derived along a single direction is consequently not representative for the entire stellar surface. If rotational and magnetic particularities of the star are not taken into account, the determined mass loss rates (and derived empirical relationships) can be subject to larger uncertainties. Since the difference of the latitudinal variation between a Polar Spot and a Latitudinal Belt distribution is about one and half magnitudes, a considerable reduction of these uncertainties could be achieved by verifying which of the two cases describes the actual magnetic field distributions of rapidly rotating stars more accurately. But for this, the yet small number of ZDI observations and field extrapolations has to be increased considerably. The X-ray luminosities of the stars considered by Wood et al. (2002) are below the X-ray saturation limit (Stauffer et al. 1997) and the objects thus likely moderate rotators with rotation periods longer than about $2 \mathrm{~d}$. The possible inaccuracies of the ram pressure should accordingly be less than an order of magnitude, and the uncertainty of the mass loss rates due to the latitudinal variation less then a factor of $\sim 3$. For stars of moderate rotation rate the linear empirical relationship between the stellar X-ray emission and mass loss rate should therefore hardly be affected. Due to the increasing latitudinal variation with rotation rate, the scatter of future observations of more rapidly rotating stars (with higher $\mathrm{X}$-ray fluxes) around the predicted linear relationship is however expected to increase. The conclusion of Wood et al., that the mass loss rates of younger and more rapidly rotating stars is up to several orders of magnitude larger than in the case of the Sun, confirms the mass loss rates of rapid rotators determined in the framework of the WD approach.

In their investigation of properties of stellar magnetic fluxes and magnetised winds, Schrijver et al. (2003) combine observational stellar data in a chain of arguments, which involves the functional dependence of the (effective) Alfvénic radius on the stellar rotation rate. Since in the framework of the present wind model, the difference between the effective Alfvénic radius and its respective WD value hardly depends on the rotation rate, the impact of non-uniform field distributions on their work is expected to be marginal. In particular since their analyses is constrained to moderately active, slowly rotating stars, whose magnetic flux is typically located at low latitudes, in which case the effective Alfvénic radii are within $\sim 10 \%$ comparable to the WD value. However, as in more rapidly rotating stars magnetic flux is also found at intermediate and high latitudes, the transition between different latitude-dependent field distributions implies an additional dependence of the Alfvénic radius on the rotation rate, which would have to be taken into account. The explicit functional dependence has to be determined observationally (e.g. Strassmeier 2002) or through numerical simulations (e.g. Schüssler et al. 1996; Granzer et al. 2000).

In addition to the surface magnetic field distribution, the loss rates of magnetised winds depend significantly on coronal magnetic field topologies. Theoretical models indicate that the mass and AM loss rates of a multi-component corona consisting of both wind and dead zones (i.e., open and closed field regions, respectively) are considerably smaller than according to the WD model, since in addition to the confinement of coronal plasma in magnetic loops dead zones also alter the flow structure in adjacent wind regions (Priest \& Pneuman 1974; Mestel $\&$ Spruit 1987). Observations in the case of the Sun however indicate that the mass loss rate arising from the slow solar wind, traced back to closed equatorial field structures, is within a 
factor of two comparable with the mass loss rate of the fast solar wind, which originates from open coronal holes (Wang 1998).

Simplified approaches to the wind model like the one used here typically ignore the non-linear influence of the trans-field component so that the resulting loss rates are subject to inaccuracies. A comparison between in situ measurements of the solar wind by the Helios spacecrafts and respective WD-based estimates however showed that this basic model is an adequate description of the physical interaction coupling the rotation of a star with its associated wind, insofar as simple magnetic stresses are taken as the principal physical mechanism (Pizzo et al. 1983); for a comparison between out-of-eclipse measurements by the Ulysses spacecraft with a self-consistent model approach see, for example, Sauty et al. (2005). Furthermore, investigations of the rotational evolution of stars, which make use of magnetic braking timescales estimated according to WD-like wind models, yield rotational histories which are consistent with observed distributions of stellar rotation rates in young open clusters of different age (e.g. Solanki et al. 1997). Whereas multi-dimensional MHD-simulations solve the transfield component of the equation of motion self-consistently (e.g., Sakurai 1985, 1990; Keppens \& Goedbloed 2000), in the present work the poloidal component of the magnetic and velocity field are taken to be radial. The numerical simulations show that at large distances from the star magnetic field lines do not remain radial but tend to be deflected away from the equatorial plane to higher latitudes, leading to a collimation of the wind around the pole (Tsinganos \& Bogovalov 2000; Okamoto 2000). The influence of this phenomenon on the mass and AM loss rates is not clear, since respective investigations are focused on the detailed wind structure of individual illustrative cases like the Sun. The combination of multi-dimensional MHD-simulations with more appropriate field distributions is desirable, although the large numerical demand makes such modelling more challenging for stellar rotational evolution studies, where a large number of simulations is required to determine the relative impact of the evolving stellar structure, internal AM transport, dynamo efficiency, dynamo saturation, and surface magnetic field distributions on the stellar rotation (e.g. Holzwarth \& Jardine 2005).

\section{Conclusion}

The frequently observed non-uniform surface magnetic fields of active stars have a remarkable influence on stellar mass and AM loss rates. Concentrations of magnetic flux at high latitudes in the form of polar spots reduce the AM loss rate and effective Alfvénic radius up to $60 \%$ and $25 \%$, respectively, with respect to the approach of Weber \& Davis (1967). The large deviations make them important ingredients for studies considering the rotational evolution of stars, implying considerable consequences for the distribution of stellar rotation rates. The gathering of open magnetic flux in the form of a Latitudinal Belt has in contrast a strong impact on the error margins of empirical stellar mass loss rates derived using the ram pressure of stellar winds. In particular in the regime of rapidly rotating stars, the strong latitude-dependent magneto-centrifugal driving causes latitudinal variations of the terminal wind velocity and ram pressure which span more than two orders of magnitude. These examples show that the non-uniformity of surface magnetic fields represents an essential stellar property, whose neglect entails large uncertainties of observational and theoretical results.

The loss rates and effective Alfvénic radii resulting from different non-uniform surface magnetic fields are found to cover a large range of values, although the total magnetic flux of each field distribution is the same. This shows that the classification of stellar magnetic fields through a single, allegedly characteristic, field strength (e.g. the peak- or surface-averaged field strength) is considered to be inapplicable. Instead, possible non-uniformities of the surface magnetic fields have to be taken into account, based either on empirical relationships or in the framework of theoretical constraints. In view of the importance of the actual location of open magnetic flux, this makes an increase of the yet small number of combined Zeeman-DI observations and reconstructions of global stellar magnetic field topologies highly eligible.

Acknowledgements. The author would like to thank M. Jardine and S. Jeffers for valuable discussions and comments, and the anonymous referee for suggestions, which helped to improve the paper. This research was funded by a PPARC standard grand (PPA/G/S/2001/00144).

\section{Appendix A: Polytropic wind model}

The stellar wind is taken to be an ideal plasma with vanishing viscosity and infinite conductivity. Its structure is determined by the stationary equation of motion, and subject to a polytropic relation between the thermal pressure, $p$, and density, $\rho$. The polytropic index, $\Gamma$, quantifies the heating of the wind; a value close to one implies a nearly isothermal wind flow and a value close to the ratio of specific heats an almost adiabatic expansion.

\section{A.1. Basic relations and invariants}

In a reference system co-rotating with the stellar surface a stationary plasma flow obeys the relation $\boldsymbol{v}_{\mathrm{co}}=\kappa \boldsymbol{B} / \rho$, where $\boldsymbol{v}_{\mathrm{co}}$ is the flow velocity, $\boldsymbol{B}$ the magnetic field, and $\kappa$ the ratio between the mass flux and the magnetic flux; along a magnetic field line the latter is constant. The flow velocity in the rest frame of reference is given by

$\boldsymbol{v}=\boldsymbol{v}_{\mathrm{co}}+\boldsymbol{\Omega} \times \boldsymbol{r}=\kappa \frac{\boldsymbol{B}}{\rho}+\boldsymbol{\Omega} \times \boldsymbol{r}$,

where $\boldsymbol{r}$ is the vector pointing from the centre of the star to the location of a gas element, and $\boldsymbol{\Omega}$ a vector parallel to the stellar rotation axis, whose modulus, $\Omega$, is the stellar rotation rate. The wind is assumed to be axi-symmetric with respect to the rotation axis, $\boldsymbol{e}_{\Omega}=\boldsymbol{e}_{z}$, that is all derivatives in azimuthal direction, $\boldsymbol{e}_{\phi}$, vanish.

Owing to the rotational symmetry, the azimuthal component of the equation of motion can be written in the form

$\boldsymbol{v} \cdot \nabla\left(v_{\phi} \varpi\right)=\frac{1}{4 \pi \rho} \boldsymbol{B} \cdot \nabla\left(B_{\phi} \varpi\right)$, 
with $\varpi=r \sin \theta$. Substituting Eq. (A.1) into Eq. (A.2) yields that the specific angular momentum (per unit mass),

$L=v_{\phi} \varpi-\frac{1}{4 \pi \kappa} B_{\phi} \varpi$,

of plasma escaping along an open magnetic field line is constant. In addition to the common specific angular momentum of a moving gas element, $\propto \boldsymbol{r} \times \boldsymbol{v}$, this quantity also comprises the tension of the magnetic field bent by the gas motion ${ }^{1}$. Following Eq. (A.3), the azimuthal flow velocity is written in the form

$v_{\phi}=\frac{L-\frac{\rho}{4 \pi \kappa^{2}} \varpi^{2} \Omega}{\varpi\left(1-\frac{\rho}{4 \pi \kappa^{2}}\right)}$.

For this expression to be finite at the Alfvénic point, $\left(\varpi_{\mathrm{A}}, \rho_{\mathrm{A}}=\right.$ $4 \pi \kappa^{2}$ ), the specific angular momentum has to be,

$L=\Omega \varpi_{\mathrm{A}}^{2}=$ const.

The Alfvénic point marks the location along a magnetic field line where the Alfvénic Mach number is one (i.e. $v_{\mathrm{A}}=$ $\left.B_{\mathrm{A}} / \sqrt{4 \pi \rho_{\mathrm{A}}}\right)$, and the flow velocity changes from sub-Alfvénic to super-Alfvénic values.

Since (in the rest frame of reference) the flow velocity of the wind is oblique to the magnetic field, the resulting Poynting flux leads to an energy transfer,

$\frac{1}{4 \pi \rho} \boldsymbol{v} \cdot[(\nabla \times \boldsymbol{B}) \times \boldsymbol{B}]=\Omega \boldsymbol{v} \cdot \nabla\left(v_{\phi} \varpi\right)$,

from the stellar rotation into the gas motion via the magnetic field, which contributes to the wind acceleration in the form of a magneto-centrifugal driving. Taking Eq. (A.6) and the polytropic gas relation into account, the energy function,

$H=\frac{v^{2}}{2}+\frac{\Gamma}{\Gamma-1} \frac{p}{\rho}+\Psi-v_{\phi} \Omega \varpi$,

with $\Psi$ being the gravitational potential, has to be constant along a magnetic field line,

$H=E=$ const.

The four terms of Eq. (A.7) are (in given order) the kinetic, the thermal, the gravitational, and the magneto-centrifugal contribution to the specific energy of the outflow. The Eqs. (A.7) and (A.8) follow from the component of the equation of motion tangential to the wind flow and correspond to the Bernoulli integral. The two constants, Eqs. (A.5) and (A.8), are a direct consequence of the invariance of the problem against azimuthal and temporal translations (i.e. axial symmetric and stationary flow). For a more detailed description of the theory of magnetised winds see Mestel (1999); Lamers \& Cassinelli (1999).

A full treatment of the stellar wind problem requires the self-consistent solution of its trans-field component, which

\footnotetext{
${ }^{1}$ Considering the physical connection of Eq. (A.3) with the vorticity and current density, Goedbloed et al. (2004) use for the quantity $L$ the nomenclature poloidal vorticity-current density stream function and the symbol $K$ to distinguish it from the specific angular momentum of gas flows in hydrodynamics.
}

determines the poloidal field structure of the stellar wind. This aspect involves the treatment of a non-linear partial differential equation, which requires more sophisticated theoretical and/or numerical methods (e.g., Sakurai 1985; Vlahakis \& Tsinganos 1998; Keppens \& Goedbloed 2000). More detailed investigations show that at large distances from the star, in the asymptotic regime, magnetic field lines are deflected toward the pole (Heyvaerts \& Norman 1989; Tsinganos \& Bogovalov 2000; Okamoto 2000). In the present work the trans-field component is neglected and the poloidal field component instead a priori taken to be radial (cf. Yeh 1976). Consequently, the field lines form spirals around the rotation axis of the star, which are located on conical surfaces with constant opening angle. This approach is a simple generalisation of the Weber \& Davis (1967) method in the sense that the individual boundary conditions and properties of open field lines are explicitly taken into account at all latitudes, although their mutual interaction is suppressed. Due to the latter simplification the absolute values should however only be considered as lowest-order estimates.

\section{A.2. Non-dimensional wind solutions}

The determination of wind solutions follows the method of Sakurai (1990); see also Sakurai (1985, Sect. 2). Using the nondimensional variables ${ }^{2}$

$x=\frac{r}{r_{\mathrm{A}}}\left(=\frac{\varpi}{\varpi_{\mathrm{A}}}\right), \quad y=\frac{\rho}{\rho_{\mathrm{A}}}, \quad z=\frac{v_{r}}{v_{r, \mathrm{~A}}}$,

Eq. (A.7) is written in dimensionless form,

$$
\begin{aligned}
\tilde{H}= & H \frac{r_{\mathrm{A}}}{G M_{*}}=\frac{K_{\beta}}{2} z^{2}+\frac{K_{\Omega}}{2}\left[\left(\frac{x z\left(1-x^{2}\right)}{1-x^{2} z}\right)^{2}-x^{2}\right] \\
& -\frac{1}{x}+\frac{K_{\mathrm{T}}}{\Gamma-1}\left(x^{2} z\right)^{1-\Gamma},
\end{aligned}
$$

with the non-dimensional parameters

$$
K_{\mathrm{T}}=\frac{\Gamma}{2 x_{\mathrm{Pa}}} \frac{1}{y_{0}^{\Gamma-1}}, \quad K_{\Omega}=\frac{1}{x_{\Delta g}^{3}}, \text { and } \quad K_{\beta}=\frac{x_{0}^{4} y_{0}}{\beta_{0} x_{\mathrm{Pa}}} .
$$

Quantities with index " 0 " refer to values at the reference level, $r_{0}$.

The parameter $K_{\Omega}$ is the only quantity that mediates a dependence of the wind on both the stellar rotation rate and latitude. In the equatorial plane the magneto-centrifugal driving of the stellar wind is quantified through the co-rotation radius, $r_{\mathrm{co}}=\sqrt[3]{G M_{*} / \Omega^{2}}$, which specifies the distance from the star where the inward directed gravitation is balanced by the outward directed centrifugal force. Here, the magnetic field lines outside the equatorial plane are situated on coni with constant opening angles. Hence the latitude- and rotation-dependent radius

$r_{\Delta g}=\sqrt[3]{\frac{G M_{*}}{\Omega^{2}}} \sin ^{-2 / 3} \theta$

${ }^{2}$ Since along a magnetic field line the mass flux per solid angle, $F=r^{2} \rho v_{\mathrm{r}}$, is constant, the relation $x^{2} y z=1$ holds. In contrast to Sakurai (1985), here the flow velocity is used as an independent variable instead of the density. 
denotes the point beyond which in radial direction, $\boldsymbol{e}_{\mathrm{r}}$, the centrifugal acceleration of the gas, $\boldsymbol{a}_{\mathrm{c}}$, outbalances the gravitational acceleration, $\boldsymbol{g}$, that is $\left(\boldsymbol{g}+\boldsymbol{a}_{\mathrm{c}}\right) \cdot \boldsymbol{e}_{\mathrm{r}}=0$. The parameters $K_{\mathrm{T}}$ and $K_{\beta}$ depend on the boundary conditions $x_{0}$ and $y_{0}=1 /\left(x_{0}^{2} z_{0}\right)$, determined at the reference radius, $r_{0}$. There, the base temperature, $T_{0}$, of the wind defines the Parker radius,

$r_{\mathrm{Pa}}=\frac{G M_{*}}{2 c_{\mathrm{I}, 0}^{2}}=\frac{G M_{*} \mu}{2 \mathfrak{R}} \frac{1}{T_{0}}$,

where $c_{\mathrm{I}, 0}$ is the (isothermal) sound speed, and $\mu$ the mean atomic weight of the plasma. The dependence of the wind on the magnetic field strength enters through the parameter

$\beta_{0}=\frac{8 \pi p_{0}}{B_{0}^{2}}$

which denotes the ratio between the gas pressure and the magnetic pressure at the base of the wind.

Since $\tilde{\mathcal{H}}=\tilde{E}=$ const., a non-dimensional wind solution, $z(x)$, is represented in the $(x, z)$-plane by an isoline. Along this trajectory the total differential of the energy function,

$\mathrm{d} \tilde{H}=\left(\frac{\partial \tilde{H}}{\partial x}\right)_{z} \mathrm{~d} x+\left(\frac{\partial \tilde{H}}{\partial z}\right)_{x} \mathrm{~d} z$

vanishes, so that the variation of the flow velocity in radial direction is determined by the differential equation

$\frac{\mathrm{d} z}{\mathrm{~d} x}=-\frac{\left(\frac{\partial \tilde{H}}{\partial x}\right)_{z}}{\left(\frac{\partial \tilde{H}}{\partial z}\right)_{x}}$

To prevent this derivative from becoming infinite at points where the numerator vanishes, the denominator simultaneously has to be zero. The condition

$\left(\frac{\partial \tilde{H}}{\partial z}\right)_{x}=\left(\frac{\partial \tilde{H}}{\partial x}\right)_{z}=0$

defines two locations along the trajectory, which are usually referred to as the slow and fast critical points, $\left(x_{\mathrm{s}}, z_{\mathrm{s}}\right)$ and $\left(x_{\mathrm{f}}, z_{\mathrm{f}}\right)$, respectively. Since both points are situated on the same energy level, the condition

$\tilde{\mathcal{H}}\left(x_{\mathrm{s}}, z_{\mathrm{s}}\right)=\tilde{\mathcal{H}}\left(x_{\mathrm{f}}, z_{\mathrm{f}}\right)=\tilde{\mathcal{E}}$

holds. The five non-linear algebraic Eqs. (A.17), for the slow and fast critical points, and (A.18) are used to determine sets of non-dimensional wind solutions $\left(x_{\mathrm{s}}, z_{\mathrm{s}}, x_{\mathrm{f}}, z_{\mathrm{f}}, K_{\beta}\right)$ as a function of the two remaining parameters $K_{\mathrm{T}}$ and $K_{\Omega}$. These solutions are as yet independent of specific boundary conditions; the only physical quantity explicitly entering Eq. (A.10) is the polytropic index. For a given value of $\Gamma$, a look-up table is precalculated to speed up the following determination of dimensional wind solutions, which depend on the boundary conditions at the reference level.

\section{A.3. Dimensional boundary conditions}

In addition to the stellar rotation rate, $\Omega$, a dimensional wind solution requires the definition of the temperature, $T_{0}$, the density, $\rho_{0}$, and the radial magnetic field strength, $B_{r, 0}$ at the reference level, $r_{0}$. The link between these dimensional boundary conditions and the dimensionless wind solutions above is established through a combination of Eqs. (A.11),

$K_{\mathrm{T}} K_{\Omega}^{\Gamma-4 / 3} K_{\beta}^{\Gamma-1}=\frac{\Gamma}{2}\left(\frac{r_{\Delta g}}{r_{0}}\right)^{4-3 \Gamma}\left(\frac{r_{\mathrm{Pa}}}{r_{0}}\right)^{-\Gamma} \beta_{0}^{1-\Gamma}$,

to eliminate the yet unknown Alfvénic radius, $r_{\mathrm{A}}$, and density, $\rho_{\mathrm{A}}$, as well as the energy relation

$\frac{\tilde{\mathcal{E}}}{K_{\Omega}^{1 / 3}}=-\frac{r_{\Delta g}}{r_{0}}\left[1+\frac{1}{2}\left(\frac{r_{\Delta g}}{r_{0}}\right)^{-3}-\frac{1}{2} \frac{\Gamma}{\Gamma-1}\left(\frac{r_{\mathrm{Pa}}}{r_{0}}\right)^{-1}\right]$.

The latter is determined at the reference level, where the specific kinetic energy of the wind flow is assumed to be negligible compared with the thermal and centrifugal energy. For a given set of boundary conditions, the right sides of Eqs. (A.19) and (A.20) are constant and the solution found by an appropriate choice of $K_{\mathrm{T}}$ and $K_{\Omega}$; note that $K_{\beta}$ and $\tilde{\mathcal{E}}$ are both implicit functions of these independent variables. Once a nondimensional wind solution has been found, the Alfvénic radius and density are determined by back-substitution of the parameters $K_{\mathrm{T}}, K_{\Omega}$, and $K_{\beta}$.

\section{A.4. Terminal wind velocity}

The terminal wind velocity, $z_{\infty}$, is determined using Eq. (A.10) in the limit of large distances from the star $(x \rightarrow \infty)$, which yields the cubic equation

$z^{3}-z 2 \frac{K_{\Omega}}{K_{\beta}}\left(1+\frac{\tilde{E}}{K_{\Omega}}\right)+2 \frac{K_{\Omega}}{K_{\beta}}=0$.

The correct solution is subject to the constraint that even far away from the star the wind velocity has still to increase, that is

$\left.\frac{\mathrm{d} z}{\mathrm{~d} x}\right|_{x \rightarrow \infty} \simeq \frac{2 z^{2}}{x} \frac{K_{\mathrm{T}} y^{\Gamma-1}}{\left(z^{3} K_{\beta}-K_{\Omega}\right)}>0$.

For a stationary outflow to exist, the terminal wind velocity has to exceed a latitude-dependent minimal flow velocity,

$z_{\infty}>z_{\mathrm{M}}=\sqrt[3]{\frac{K_{\Omega}}{K_{\beta}}}=\frac{v_{\mathrm{M}}}{v_{r, \mathrm{~A}}} \sin ^{2 / 3} \theta$,

with $v_{\mathrm{M}}=\sqrt[3]{\Omega^{2} r_{\mathrm{A}}^{2} v_{r, \mathrm{~A}}}$ being the characteristic Michel velocity of the wind (Michel 1969). With a proper set of parameters $K_{\Omega}, K_{\beta}$, and $\tilde{E}$ obtained from Eqs. (A.19) and (A.20), the terminal velocity is determined from Eqs. (A.21) and (A.23).

\section{References}

Belcher, J. W., \& MacGregor, K. B. 1976, ApJ, 210, 498

Collier Cameron, A. 2001, Astrotomography, Indirect Imaging Methods in Observational Astronomy, LNP, 573, 183 
Collier Cameron, A., \& Jianke, L. 1994, MNRAS, 269, 1099

Donati, J.-F., \& Collier Cameron, A. 1997, MNRAS, 291, 1

Donati, J.-F., Semel, M., Carter, B. D., Rees, D. E., \& Collier Cameron, A. 1997, MNRAS, 291, 658

Endal, A. S., \& Sofia, S. 1981, ApJ, 243, 625

Goedbloed, J. P., Beliën, A. J. C., Van der Holst, B., \& Keppens, R. 2004, Phys. Plasmas, 11, 28

Granzer, T., Schüssler, M., Caligari, P., \& Strassmeier, K. G. 2000, A\&A, 355, 1087

Heyvaerts, J., \& Norman, C. 1989, ApJ, 347, 1055

Holzwarth, V., \& Jardine, M. 2005, A\&A, submitted

Jardine, M., Collier Cameron, A., \& Donati, J.-F. 2002, MNRAS, 333, 339

Kawaler, S. D. 1988, ApJ, 333, 236

Keppens, R., \& Goedbloed, J. P. 1999, A\&A, 343, 251

Keppens, R., \& Goedbloed, J. P. 2000, ApJ, 530, 1036

Keppens, R., MacGregor, K. B., \& Charbonneau, P. 1995, A\&A, 294, 469

Lamers, H. J. G. L. M., \& Cassinelli, J. P. 1999, Introduction to stellar winds (Cambridge University Press)

Li, J. 1999, MNRAS, 302, 203

Lima, J. J. G., Priest, E. R., \& Tsinganos, K. 2001, A\&A, 371, 240

McIvor, T., Jardine, M., Cameron, A. C., Wood, K., \& Donati, J.-F. 2004, MNRAS, 355, 1066

Mestel, L. 1968, MNRAS, 138, 359

Mestel, L. 1999, Stellar magnetism (Oxford University Press)

Mestel, L., \& Spruit, H. C. 1987, MNRAS, 226, 57

Michel, F. C. 1969 , ApJ, 158, 727

Montesinos, B., Thomas, J. H., Ventura, P., \& Mazzitelli, I. 2001, MNRAS, 326, 877
Okamoto, I. 1974, MNRAS, 166, 683

Okamoto, I. 2000, MNRAS, 318, 250

Parker, E. N. 1958, ApJ, 128, 664

Pizzo, V., Schwenn, R., Marsch, E., et al. 1983, ApJ, 271, 335

Priest, E. R., \& Pneuman, G. W. 1974, Sol. Phys., 34, 231

Sakurai, T. 1985, A\&A, 152, 121

Sakurai, T. 1990, Comp. Phys. Rep., 12, 247

Sauty, C., Lima, J. J. G., Iro, N., \& Tsinganos, K. 2005, A\&A, 432, 687

Sauty, C., Trussoni, E., \& Tsinganos, K. 2002, A\&A, 389, 1068

Schatzman, E. 1962, Ann. Astrophys., 25, 18

Schrijver, C. J., DeRosa, M. L., \& Title, A. M. 2003, ApJ, 590, 493

Schrijver, C. J., \& Title, A. M. 2001, ApJ, 551, 1099

Schüssler, M., Caligari, P., Ferriz-Mas, A., Solanki, S. K., \& Stix, M. 1996, A\&A, 314, 503

Schüssler, M., \& Solanki, S. K. 1992, A\&A, 264, L13

Solanki, S. K., Motamen, S., \& Keppens, R. 1997, A\&A, 325, 1039

Stauffer, J. R., Hartmann, L. W., Prosser, C. F., et al. 1997, ApJ, 479, 776

Strassmeier, K. G. 2002, Astron. Nachr., 323, 309

Tsinganos, K., \& Bogovalov, S. 2000, A\&A, 356, 989

Vlahakis, N., \& Tsinganos, K. 1998, MNRAS, 298, 777

Wang, Y.-M. 1998, in Cool Stars, Stellar Systems, and the Sun, ASP Conf. Ser., 154, 131

Weber, E. J., \& Davis, L. J. 1967, ApJ, 148, 217

Wood, B. E., \& Linsky, J. L. 1998, ApJ, 492, 788

Wood, B. E., Müller, H., Zank, G. P., \& Linsky, J. L. 2002, ApJ, 574, 412

Yeh, T. 1976, ApJ, 206, 768 